\title{
Erastin-induced ferroptosis causes physiological and pathological changes in healthy tissues of mice
}

\author{
JING ZHAO $^{1}$, BEN XU $^{1}$, QINGQING XIONG ${ }^{1}$, YUNFEI FENG ${ }^{2}$ and HUAHUA DU ${ }^{1}$ \\ ${ }^{1}$ Key Laboratory of Animal Feed and Nutrition of Zhejiang Province, College of Animal Science, \\ Zhejiang University, Hangzhou, Zhejiang 310058; ${ }^{2}$ Department of Endocrinology and Metabolism, \\ The First Affiliated Hospital of Medical School of Zhejiang University, Hangzhou, Zhejiang 310000, P.R. China
}

Received May 7, 2021; Accepted July 20, 2021

DOI: $10.3892 / \mathrm{mmr} .2021 .12352$

\begin{abstract}
Ferroptosis is a non-apoptotic form of cell death that relies on iron and lipid peroxidation, which is associated with multiple pathological processes in several diseases. Erastin is a small molecule capable of initiating ferroptotic cell death in cancer cells, which has shown great potential for cancer therapy. However, the physiological and pathological role of erastin-induced ferroptosis on healthy tissues has not been well characterized. The present study intraperitoneally injected erastin into healthy mice to detect the metabolic changes of several tissues of mice. Erastin injection induced typical characteristics of ferroptosis with higher level of serum iron and malondialdehyde and lower level of glutathione and glutathione peroxidase 4 protein. Erastin injection enhanced iron deposition in the brain, duodenum, kidney and spleen of mice. Erastin-induced ferroptosis altered the blood index values, causing mild cerebral infarction of brain and enlarged glomerular volume of kidney. It also promoted the growth of duodenal epithelium with thicker, longer and denser villi in erastin-treated mice. The findings provided evidence that erastin induced ferroptosis and caused pathological changes in healthy tissues of mice. This suggested that the anti-tumor drug erastin was somewhat toxic to healthy tissues.
\end{abstract}

\section{Introduction}

Ferroptosis is an iron-dependent, caspase-independent and non-apoptotic regulated cell death, which is induced by excessive accumulation of lipid peroxide (1). Cell death is believed to be the result of one of two distinct processes; programmed

Correspondence to: Dr Huahua Du, Key Laboratory of Animal Feed and Nutrition of Zhejiang Province, College of Animal Science, Zhejiang University, 866 Yuhangtang Road, Hangzhou, Zhejiang 310058, P.R. China

E-mail: huahuadu@zju.edu.cn

Key words: erastin, ferroptosis, iron deposition, lipid peroxidation, morphology changes cell death (apoptosis, pyroptosis, autophagy and ferroptosis) or uncontrolled cell death (necrosis and oncosis) (2). Ferroptosis has been distinguished from other types of cell death, such as apoptosis, autophagic cell death and necroptosis, at the cell morphology, genetic and biochemistry levels (1). Iron accumulation and an increase in lipid peroxidation, determined by high levels of malondialdehyde (MDA) content, are typical characteristics of ferroptosis (3). The upregulation of prostaglandin-endoperoxide synthase 2 (Ptgs2), a peroxidase, is the only downstream marker of ferroptosis (3). Ferroptosis has been reported to participate in various pathological processes, including cancer cell death (4), neurotoxicity (5), acute kidneys failure (6), hepatotoxicity (7) and intestinal ischemia/reperfusion injury (8).

Ferroptosis can be elicited by pharmacologically inhibiting the cysteine/glutamate antiporter, system $\mathrm{Xc}^{-}$, in cancer cells (3). Erastin was first identified in 2003 as a ferroptosis inducer for screening a cancer drug to be selectively lethal to oncogenic RAS mutant cell lines (9). This compound could inhibit solute carrier family 7 member 11 (SLC7A11), part of a heterodimer named system $\mathrm{Xc}^{-}$. System $\mathrm{Xc}^{-}$in the cell membrane is responsible for cellular cystine uptake to synthesize glutathione (10). Since cysteine is necessary for the synthesis of the antioxidant glutathione (GSH), erastin leads to a depletion of intracellular GSH and inactivation of glutathione peroxidase 4 (GPX4), increased lipid reactive oxygen species (ROS) formation and peroxidation, thus triggering ferroptosis (11). Erastin-induced ferroptosis shows great potential for cancer therapy (12). However, the physiological role and pathological effect of erastin-induced ferroptosis on healthy tissues has not been well characterized.

Erastin is already widely used to induce ferroptosis in cancer cells in vitro (13). The aim of the present study was to identify the side effects of erastin-induced ferroptosis on healthy tissues in vivo. A mouse model of erastin-induced ferroptosis was established and it was found that erastin-induced ferroptosis caused mild cerebral infarction of the brain, duodenal epithelium hyperplasia and glomeruli enlargement. The present study provided some evidence of pathological changes induced by erastin treatment and it is hoped that further studies will look at the prevention of such side effects of erastin when used for cancer therapy. 


\section{Materials and methods}

Animals. A total of 12 male C57BL/6 mice (weight, 20-21 g; age, 8 weeks) were purchased from SLAC Laboratory Animal Co., Ltd. All mice were housed in standard plastic cages and contained food and water ad libitum at Zhejiang University at $24^{\circ} \mathrm{C}$ with a 12 -h light/dark cycle. The experimental procedures for the mice were approved by Animal Ethics Committee of Zhejiang University (approval no. 20077). In accordance with a previous study by Huo et al (14), mice were intraperitoneal injected with $25 \mathrm{mg} / \mathrm{kg}$ body weight of erastin (Selleck Chemicals; $\mathrm{n}=6)$ or solvent $(5 \%$ DMSO $+40 \%$ PEG400 $+5 \%$ Tween $-80+50 \%$ physiological saline; $n=6$ ) for 2 days at 12 -h intervals. Mice were anesthetized via an intraperitoneal injection of avertin $(500 \mathrm{mg} / \mathrm{kg}) 6 \mathrm{~h}$ after the last injection. Following anesthesia, peripheral blood ( $0.5 \mathrm{ml}$ per mouse) was drawn from retroorbital plexus once by inserting a micro-hematocrit capillary tube (cat. no. HCH-42A2502; Kimble Chase Life Science and Research Products LLC) into the venous sinus behind the eyeball and was used to determine erythrocyte parameters and serum iron level by automatic SYSMEX F820 analyzer (Sysmex Corporation). Total iron binding capacity was calculated by measuring serum iron and serum unsaturated iron-binding capacity and summing these values. Animals were sacrificed following blood collection by cervical dislocation. Tissue samples from duodenum, kidney, liver, spleen, testis and brain were frozen in liquid nitrogen and held at $-80^{\circ} \mathrm{C}$ for analysis.

Reverse transcription-quantitative (RT-q) PCR. Total RNA was isolated from intestine, kidney, liver, spleen and testis tissues, respectively, using TRIzol ${ }^{\circledR}$ (Invitrogen; Thermo Fisher Scientific, Inc.). RNA quantity and purity were determined using a NanoDrop 2000 spectrophotometer (Thermo Fisher Scientific, Inc.). For cDNA synthesis, 2,000 ng RNA was reverse transcribed using Hifair ${ }^{\circledR}$ III 1st Strand cDNA Synthesis SuperMix for qPCR (Shanghai Yeasen Biotechnology Co., Ltd.). qPCR was conducted on a StepOne Real-Time PCR System (ABI StepOnePlus, Applied Biosystems; Thermo Fisher Scientific, Inc.) using Hief UNICON ${ }^{\circledR}$ qPCR SYBR Green Master Mix (Shanghai Yeasen Biotechnology Co., Ltd.). The following thermocycling conditions were used for the qPCR: Initial denaturation at $95^{\circ} \mathrm{C}$ for $1 \mathrm{~min}$; followed by 40 cycles at $95^{\circ} \mathrm{C}$ for $15 \mathrm{sec}$ and $60^{\circ} \mathrm{C}$ for $1 \mathrm{~min}$. The primers were synthesized by BioSune Biotechnology. The following primers were used: Ptgs 2 forward 5'-ATACGC TGAGTGTGGTTTGC-3' and reverse 5'-CTCACTAAACCA TCCAATCGG-3'; GAPDH forward 5'-TGCGACTTCAAC AGCAACTC-3' and reverse 5'-GCCTCTCTTGCTCAGTGT CC-3'. Ptgs 2 fold changes were calculated after normalizing the change in expression of GAPDH using $2^{-\Delta \Delta C q}$ method (15). The experiments were repeated in triplicate.

Western blotting. Total protein was isolated from tissues by homogenizing in RIPA lysis buffer (Beyotime Institute of Biotechnology) containing protease inhibitor cocktail (Thermo Fisher Scientific, Inc.). The total protein content was determined using a BCA Protein Assay kit (Nanjing KeyGen Biotech Co., Ltd.). Protein samples (20 $\mu \mathrm{g} / \mathrm{lane})$ were separated by $15 \%$ SDS-PAGE and then transferred onto a PVDF membrane (MilliporeSigma). The membranes were blocked with skimmed milk for $1 \mathrm{~h}$ at room temperature, after which the membrane was incubated with the following primary antibodies overnight at $4^{\circ} \mathrm{C}$ : GAPDH (cat. no. EM1901-57; 1:10,000; HuaBio), GPX4 (cat. no. ET1706-45; 1:1,000; HuaBio) and SLC7A11 (cat. no. HA720001; 1:1,000; HuaBio). Subsequently, the membrane was washed three times with PBS and incubated with a HRP-conjugated secondary antibody (cat. no. 7074; 1:2,000; Biosharp Life Sciences) at room temperature for $1 \mathrm{~h}$. Signals were detected using chemiluminescence (ECL Plus detection system; Clinx Science Instruments Co., Ltd.) and quantified using ImageJ software (version 2.0; National Institutes of Health).

Measurement of MDA and GSH in tissues. MDA and GSH were measured using MDA and GSH Assay kit (Beijing Solarbio Science \& Technology Co., Ltd.) according to the manufacturer's instructions. The production of MDA serves as an index of lipid peroxidation and gives a pink color once reacted with thiobarbituric acid with a maximum absorption at $535 \mathrm{~nm}$. GSH assay is based on the GSH recycling system by GSH substrate (DTNB) and GSH reductase. DTNB and GSH react to generate 2-nitro-5-thiobenzoic acid which has a yellow color. Therefore, GSH concentration can be determined by measuring absorbance at $412 \mathrm{~nm}$. Optical density was measured at $532 \mathrm{~nm}$ for MDA or $412 \mathrm{~nm}$ for GSH by a microplate reader (Molecular Devices, LLC).

2,3,5-Triphenyltetrazolium chloride monohydrate (TTC) staining. The brain was sectioned coronally into 5 slices ( $2 \mathrm{~mm}$ thick) and then incubated in TTC for $10 \mathrm{~min}$ at $37^{\circ} \mathrm{C}$ and fixed in $10 \%$ buffered formalin. Survival area was defined as the ratio of TTC-stained tissue (non-ischemic) area to the entire coronal section area.

Histological examination of tissues. Cerebral, duodenal, renal, hepatic, splenic and testicular tissues were fixed in $4 \%$ paraformaldehyde overnight at $4^{\circ} \mathrm{C}$ and embedded in paraffin blocks. Sections of 5- $\mu \mathrm{m}$ were deparaffinized, rehydrated and stained with $\mathrm{H} \& \mathrm{E}$ for $1 \mathrm{~min}$ at room temperature. Determination of villus height, crypt depth was performed with $\geq 6$ villi or crypts per slide. Glomerular volume and mesangial area in each kidney section were quantified by measuring 10 non-overlapping glomeruli. For Prussian blue staining, cerebral, duodenal, renal, hepatic, splenic and testicular sections were deparaffinized and rehydrated in serial alcohols. The tissue was then incubated for $30 \mathrm{~min}$ at room temperature in an equal mixture of $2 \%$ potassium ferrocyanide and $2 \%$ hydrochloric acid. After washing, the slides were counterstained with eosin for $20 \mathrm{sec}$, dehydrated and sealed. For Masson staining, duodenal, renal and splenic sections were stained by Wiegert's iron hematoxylin for $10 \mathrm{~min}$ at room temperature, and $1 \%$ hydrochloric acid in alcohol used for differentiation at room temperature. Subsequently, sections were counterstained in Masson Ponceau acid solution for $10 \mathrm{~min}$ and sealed at room temperature. For Periodic acid-Schiff (PAS) staining, renal sections were deparaffinized and rehydrated in serial alcohols (70-100\%). Sections were treated for $15 \mathrm{~min}$ with $1 \%$ periodic acid followed by Schiff's reagent treatment for $10 \mathrm{~min}$, then stained with Gills hematoxylin for $3 \mathrm{~min}$ at room temperature. Images were 
Table I. Erastin-induced ferroptosis altered blood index values of mice.

\begin{tabular}{lccc}
\hline Parameter & Control & Erastin & P-value \\
\hline Hemoglobin $(\mathrm{g} / \mathrm{l})$ & $164.30 \pm 2.73$ & $155.50 \pm 1.56$ & 0.04 \\
Hematocrit $(\%)$ & $52.85 \pm 0.80$ & $49.55 \pm 0.52$ & 0.02 \\
Red blood cell count $\left(10^{12} / \mathrm{l}\right)$ & $10.43 \pm 0.15$ & $9.76 \pm 0.13$ & 0.01 \\
Red blood cell distribution width (\%) & $16.34 \pm 0.21$ & $15.30 \pm 0.15$ & 0.01 \\
Mean corpuscular hemoglobin (pg) & $15.77 \pm 0.08$ & $15.95 \pm 0.05$ & 0.14 \\
Mean corpuscular hemoglobin concentration (g/l) & $311.00 \pm 0.73$ & $313.80 \pm 1.11$ & 0.06 \\
Mean corpuscular volume (fl) & $50.67 \pm 0.21$ & $50.78 \pm 0.23$ & 0.74 \\
Platelet count (10\%/l) & $959.70 \pm 56.82$ & $830.50 \pm 75.40$ & 0.20 \\
Mean platelet volume (fl) & $7.13 \pm 0.07$ & $7.00 \pm 0.13$ & 0.34 \\
Plateletcrit $(\%)$ & $0.68 \pm 0.04$ & $0.58 \pm 0.05$ & 0.16 \\
Platelet distribution width (\%) & $14.73 \pm 0.02$ & $14.68 \pm 0.03$ & 0.11 \\
ALT (U/l) & $37.40 \pm 2.58$ & $33.80 \pm 3.54$ & 0.44 \\
AST (U/l) & $135.00 \pm 9.26$ & $158.00 \pm 13.94$ & 0.19 \\
\hline
\end{tabular}

captured using an Olympus NP-26 inverted light microscope (magnification, x200; Olympus Corporation). The mesangial matrix index was defined as the ratio of mesangial matrix area divided by the tuft area.

Statistical analysis. All assays were performed at least in triplicate and the values are expressed as mean \pm standard error of the mean. Statistical analysis was performed using GraphPad software (version 8.0; GraphPad Prism, Inc.) and analyzed by unpaired two-tailed Student's t-tests. $\mathrm{P}<0.05$ was considered to indicate a statistically significant difference.

\section{Results}

Erastin injection induces ferroptosis in mice. Erastin-treated mice were inactive and liked to gather together, but exhibited no significant difference in body weight compared with control mice (Fig. S1). Following erastin injection, hemoglobin, hematocrit, red blood cell count and red blood cell distribution width were all significantly $(\mathrm{P}<0.05)$ decreased in the peripheral blood of mice (Table I). Serum iron of erastin-treated mice was 6.16-fold higher $(\mathrm{P}<0.01)$ compared with control mice (Fig. 1A), as well as total iron binding capacity (Fig. 1B). Erastin injection induced a robust increase $(\mathrm{P}<0.05)$ in mRNA level of Ptgs 2 , a putative molecular marker of ferroptosis, in duodenum, kidney, liver and spleen (Fig. 1C). Compare with the control group, MDA was increased by $58 \%$ in duodenum $(\mathrm{P}<0.05), 93 \%$ in kidney $(\mathrm{P}<0.01)$ and 2.25-fold in liver $(\mathrm{P}<0.05)$ of erastin-treated mice (Fig. 1D). Erastin injection led to a decrease of GSH by $64 \%$ in duodenum $(\mathrm{P}<0.05), 34 \%$ in kidneys $(\mathrm{P}<0.01)$ and $43 \%$ in liver $(\mathrm{P}<0.05)$ (Fig. 1E). Erastin injection also inhibited SLC7A11 and GPX4 protein expression in duodenum, kidneys, liver and spleen (Fig. 1F-I). However, both proteins and mRNA level of Ptgs 2 in testis were not significantly $(\mathrm{P}>0.05)$ changed (Fig. 1C and J). Taken together, above data indicated that erastin injection successfully induced ferroptosis.

Erastin injection causes mild infarct and iron deposition in the brain. TTC staining demonstrated that erastin injection caused a mild cerebral infarction in brain, where living tissue is red while infarcted area remains white (Fig. 2A). Erastin-treated mice demonstrated $11 \%$ lower $(\mathrm{P}<0.05)$ surviving area of brain tissue compared with control mice (Fig. 2B). H\&E staining demonstrated that there was no difference between two groups in brain white matter (Fig. 2C). Prussian blue staining revealed a slight iron deposition in the brain of erastin-treated mice (Fig. 2D).

Erastin injection promotes the growth and iron deposition of duodenum. H\&E staining demonstrated that erastin-treated mice had thicker, longer and denser mucosal villi compared with the control mice (Fig. 3A). Image analysis revealed a significant increment $(\mathrm{P}<0.05)$ in the villus height $(531.9 \pm 35.48 \mu \mathrm{m})$ of erastin-treated mice, as compared with that $(435.70 \pm 12.43 \mu \mathrm{m})$ of untreated mice (Fig. 3B). Erastin injection also induced a marked reduction $(\mathrm{P}<0.05)$ in the crypt depth $(57.86 \pm 4.32 \mu \mathrm{m})$ of erastin-treated mice, as compared with that $(70.84 \pm 2.74 \mu \mathrm{m})$ of untreated mice (Fig. 3C). Therefore, the ratio of villus height to crypt depth, an indicator of absorptive function of duodenum, was upregulated by $33 \%$ in erastin-treated mice (Fig. 3D). Prussian blue staining revealed that the blue granules of hemosiderin mainly appeared on the tip of villi in erastin-treated mice (Fig. 3E). Masson staining demonstrated that there was no significant difference $(\mathrm{P}>0.05)$ in tissue fibrosis of duodenum between two groups (Fig. 3F and G).

Erastin injection increases glomerular volume of kidney. Erastin-treated mice demonstrated increased glomerular volume with dilated glomerular capillaries (Fig. 4A). The mean glomerular volume and mesangial area of erastin-treated mice were significantly $(\mathrm{P}<0.05)$ increased 1.76 - and 1.44 -fold, respectively (Fig. 4B and C), compared with those of control mice. However, the index of mesangial matrix did not change significantly ( $\mathrm{P}>0.05$; Fig. 4D). Furthermore, Masson staining demonstrated that erastin treatment did not significantly affect the degree of renal fibrosis (Fig. 4E and F). PAS staining demonstrated no significant raise of PAS-positive cells in glomerulus 
A

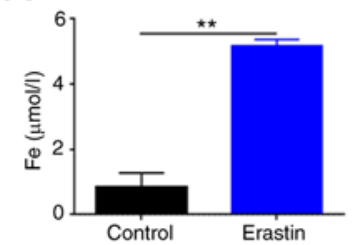

- Control
B

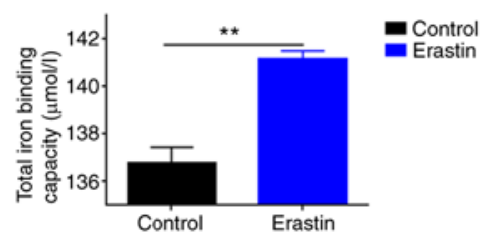

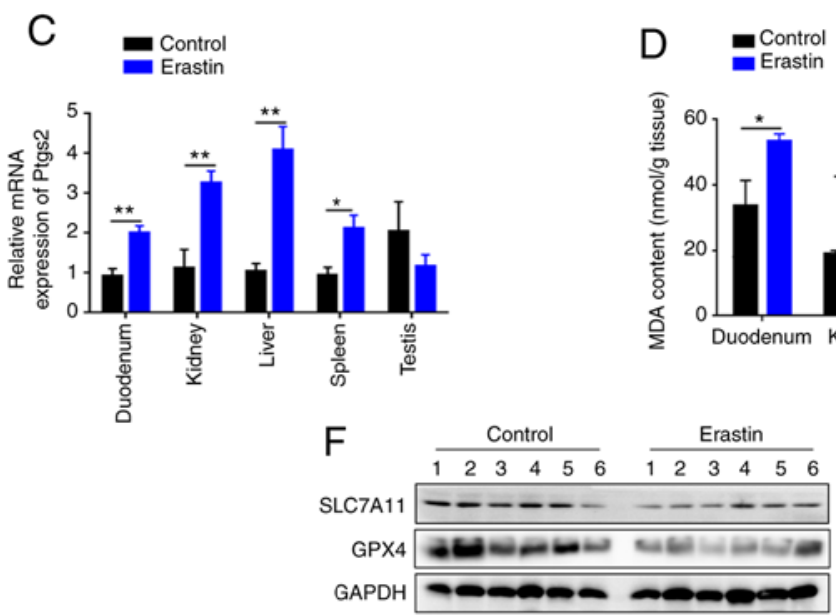
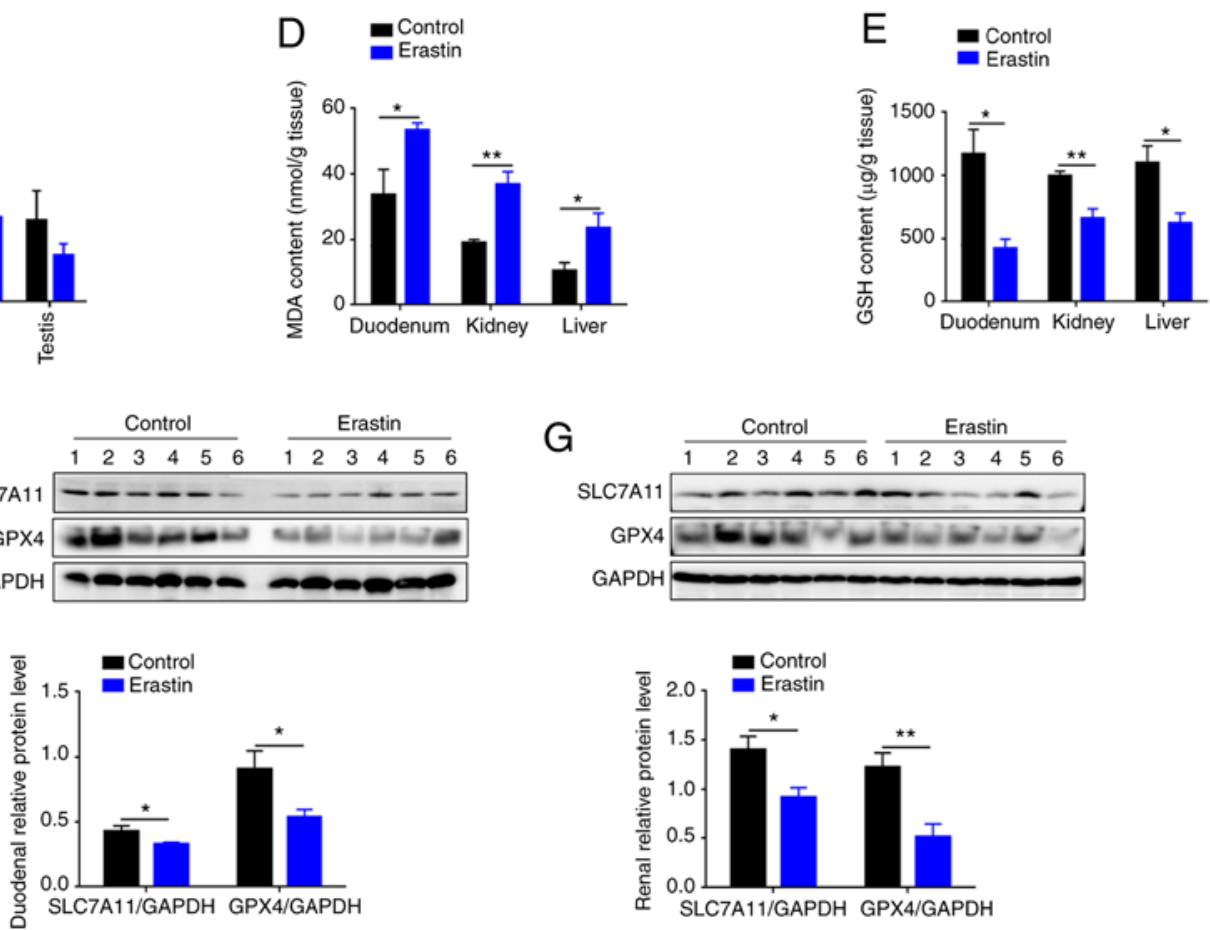
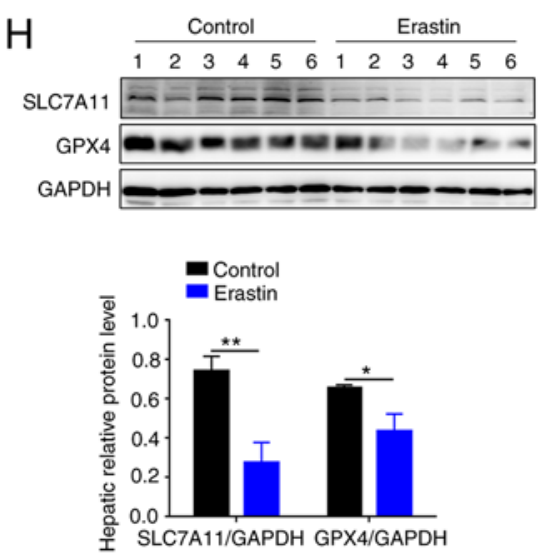

I

$$
\begin{array}{r}
\text { SLC7A11 } \\
\text { GPX4 } \\
\text { GAPDH }
\end{array}
$$$$
\text { Contro }
$$$$
\begin{array}{lllllllllllll}
1 & 2 & 3 & 4 & 5 & 6 & 1 & 2 & 3 & 4 & 5 & 6
\end{array}
$$$$
\text { GPX } 4
$$

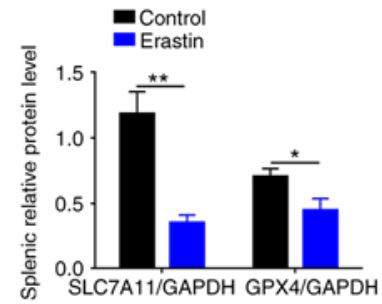

$J$
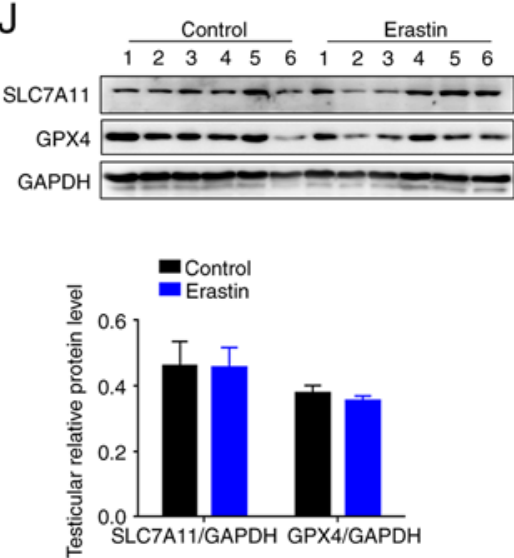

Figure 1. Erastin injection induces ferroptosis in mice. The effect of erastin injection on (A) iron level and (B) total iron binding capacity in serum. (C) Ptgs2 mRNA expression level in duodenum, kidneys, liver, spleen and testis. (D) MDA and (E) GSH content in duodenum, kidneys and liver. SLC7A11 and GPX4 protein expression levels were examined by western blotting ( $\mathrm{n}=6$ per group) in (F) duodenum, (G) kidneys, (H) liver, (I) spleen and (J) testes. Results were expressed as mean \pm standard error of the mean. ${ }^{*} \mathrm{P}<0.05,{ }^{* *} \mathrm{P}<0.01$.

and no abnormality in renal tubules in erastin-treated mice (Fig. 4G). Prussian blue staining indicated that erastin-induced ferroptosis also caused mild iron deposition in the kidneys (Fig. 4H).

Erastin injection affects iron deposition in the spleen, but no liver. In consideration of significant changes in the indicators of ferroptosis in the liver (Fig. 1C-E and H), the present study examined hepatic pathological changes by $H \& E$ staining. Notably, no significant difference was observed between the two groups. There was no necrosis, hemorrhage and inflammatory infiltration, hepatocyte apoptosis and vacuolar degeneration in the liver of erastin-treated mice (Fig. 5A). Prussian blue staining also demonstrated no apparent iron deposition in the liver of erastin-treated mice (Fig. 5B). The spleen consisted of the red pulp and white pulp and the structure of splenic corpuscles was clear in both groups (Fig. 5C). Prussian blue staining revealed the appearance of a brown substance and distinguished it from hemosiderin, which would be stained as blue granules 
A

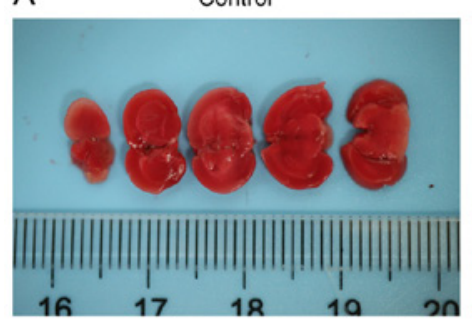

Erastin

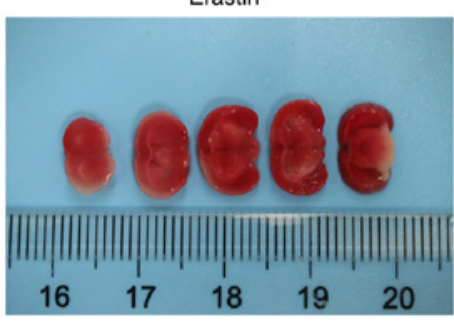

B

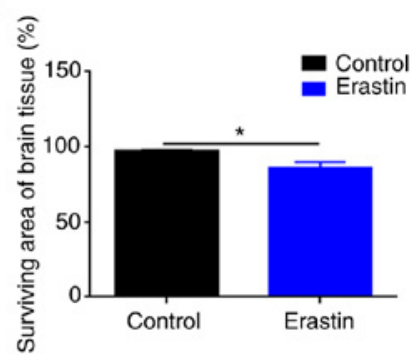

C
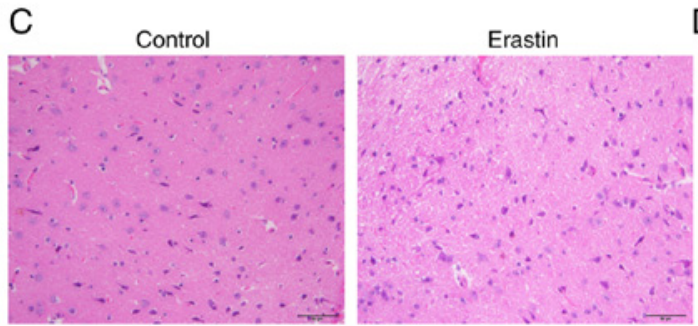

D Control
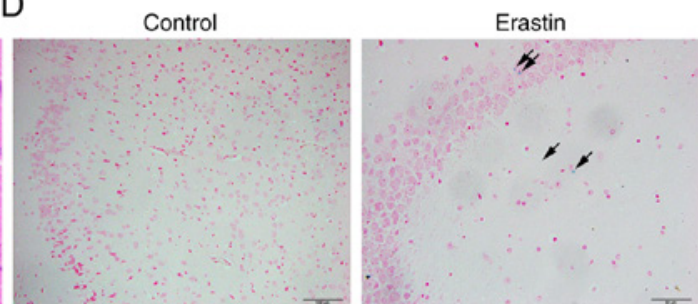

Figure 2. Erastin injection causes mild infarct and iron deposition in the brain. (A) Representative images of TTC staining and (B) quantification of surviving area. (C) hematoxylin-eosin staining and (D) Prussian blue staining of mouse brain in control and erastin groups (all magnifications, $\mathrm{x} 400$ ). Arrows indicate iron particles. Values are average means of triplicate experiments with five mice per group. Results were expressed as mean \pm standard error of the mean. "P $<0.05$.

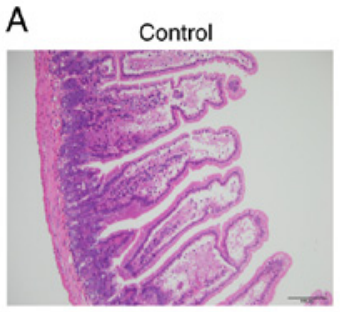

C

$\mathrm{E}$

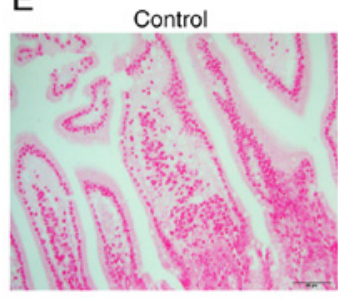

Erastin
Erastin

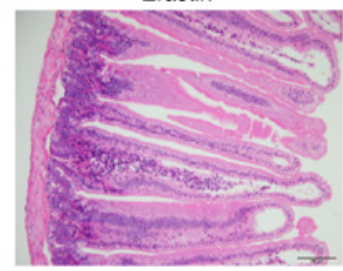

D
B Control

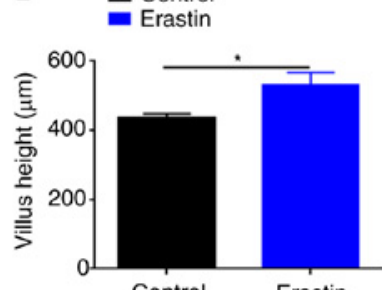

Erastin
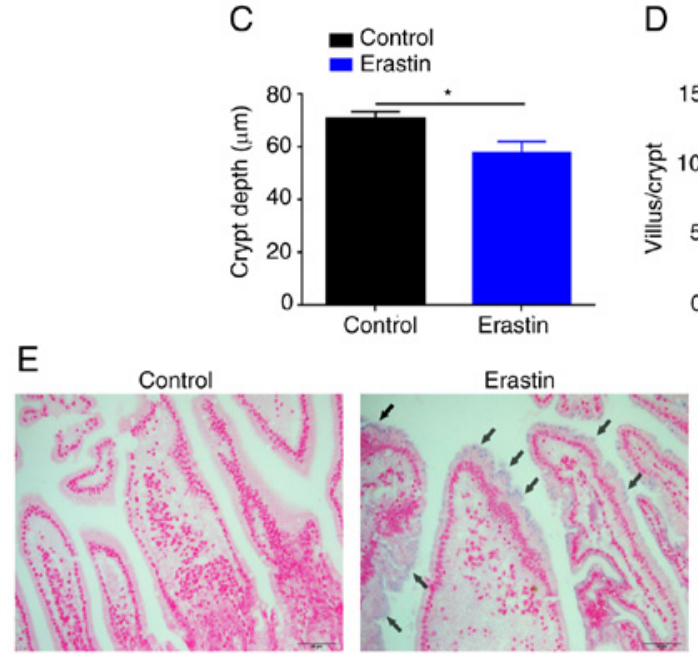

F
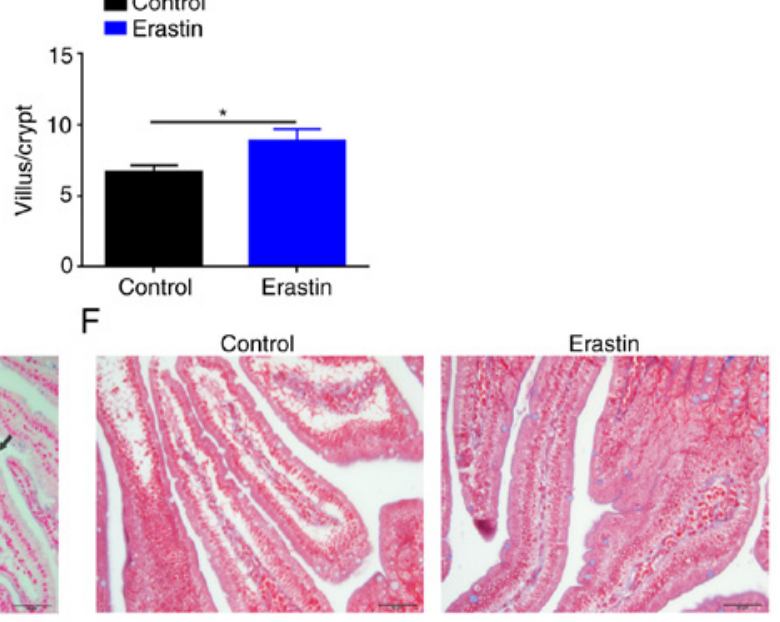

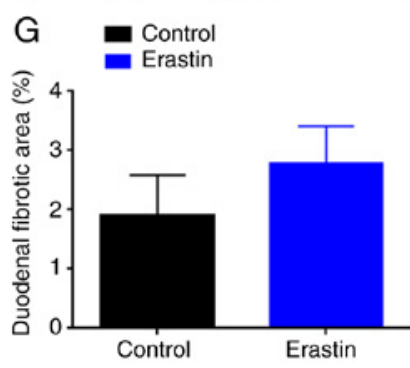

Figure 3. Erastin injection promotes duodenal growth and iron deposition. (A) Representative images of mouse duodenal (A) hematoxylin-eosin staining (magnification, $x 200$ ) and quantification of (B) villus height, (C) crypt depth and (D) villus height:crypt depth ratio in control and erastin groups. Representative images of mouse duodenal (E) Prussian blue staining (magnification, x400). Arrows indicate iron particles. (F) Masson staining (magnification, x400) and (G) quantification of duodenal fibrotic area in control and erastin groups. Values are average means of triplicate experiments with five mice per group. Results were expressed as mean \pm standard error of the mean. ${ }^{*} \mathrm{P}<0.05$. 

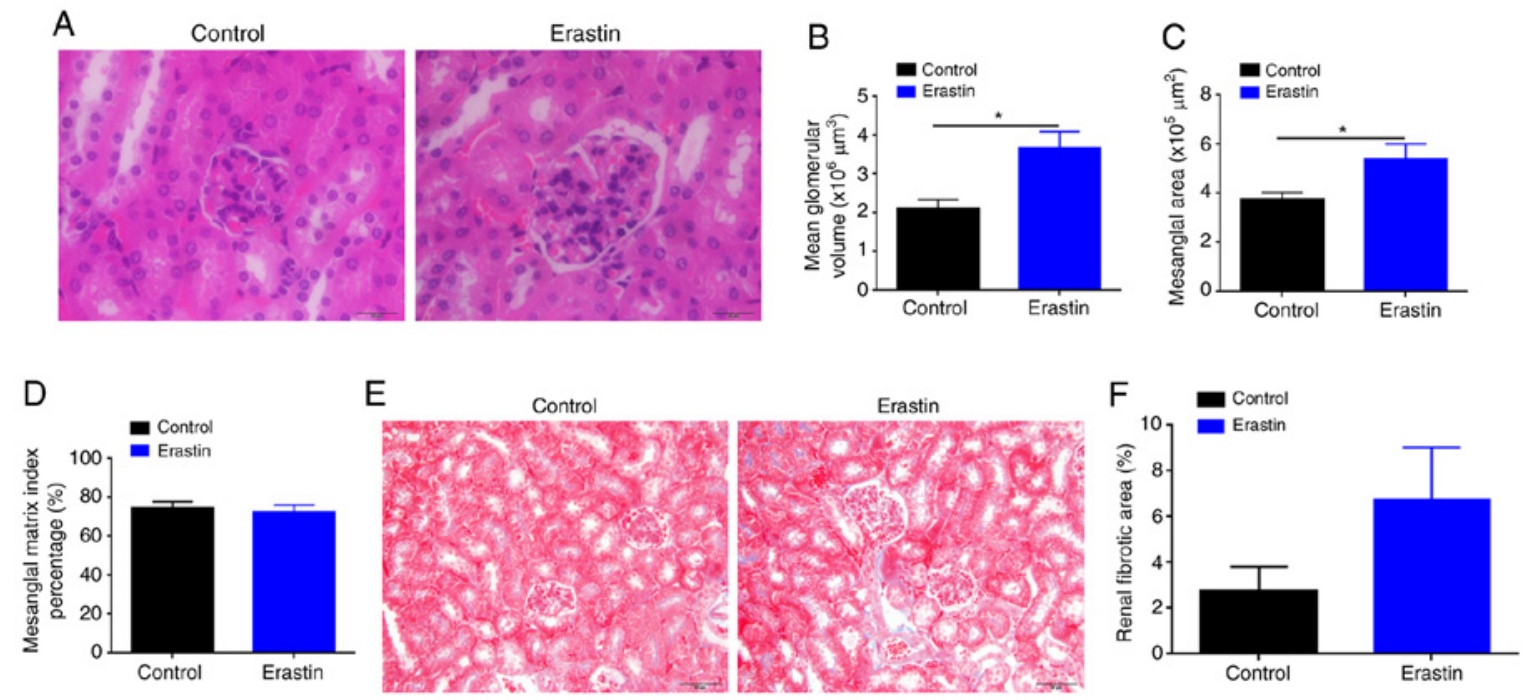

G

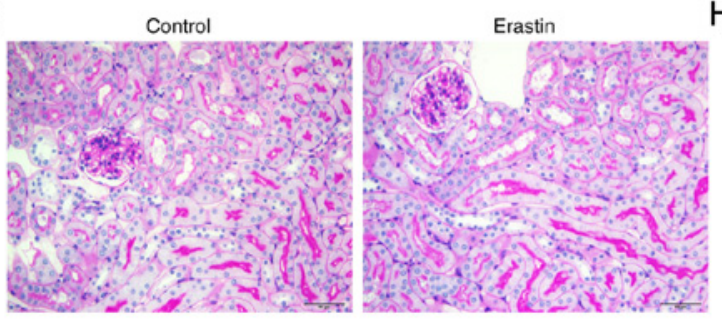

$\mathrm{H}$
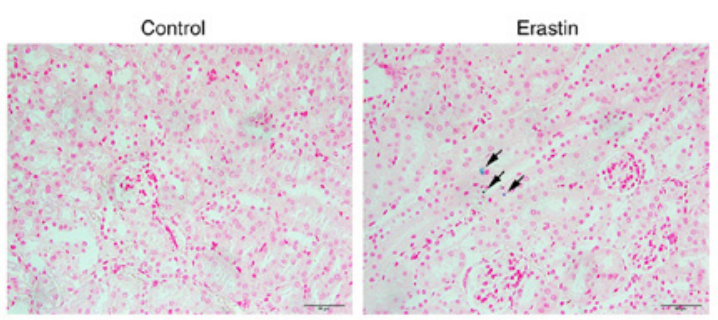

Figure 4. Erastin injection increases glomerular volume of kidney. (A) Representative images of mouse glomerular hematoxylin-eosin staining (magnification, $\mathrm{x} 1,000)$ and quantification of (B) mean glomerular volume, (C) mesangial area and (D) mesangial matrix index percentage in control and erastin groups. (E) Representative images of Masson staining (magnification, x400) and (F) quantification of renal fibrotic area. (G) PAS staining (magnification, x400) and (H) Prussian blue staining (magnification, $x 400$ ) of mouse kidneys in control and erastin groups. Arrows indicate iron particles. Values are average means of triplicate experiments with five mice per group. Results were expressed as means \pm standard error of the mean. ${ }^{*} \mathrm{P}<0.05$.
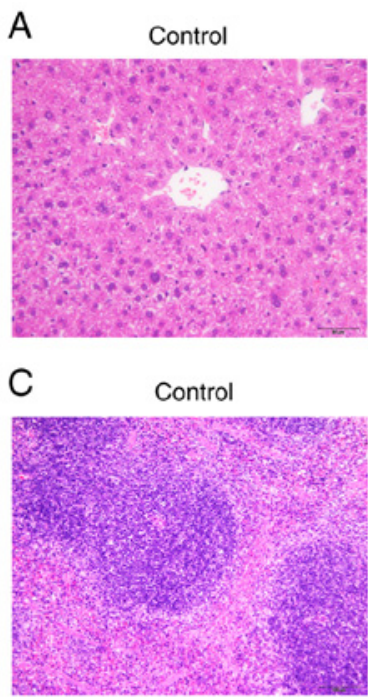
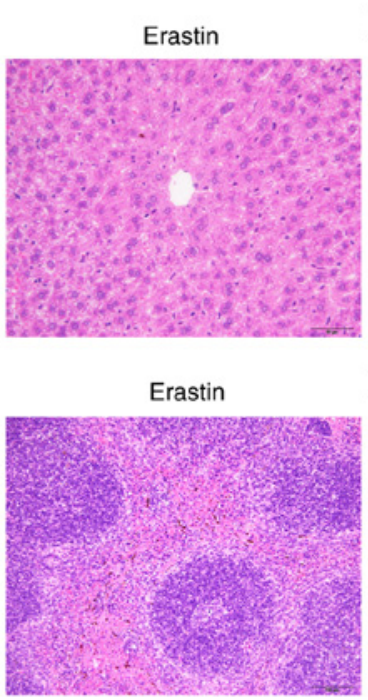

B

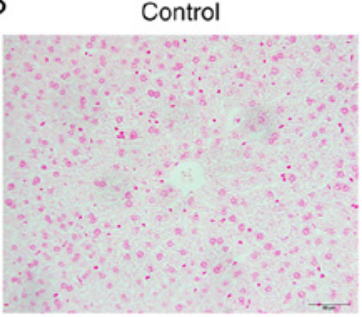

D

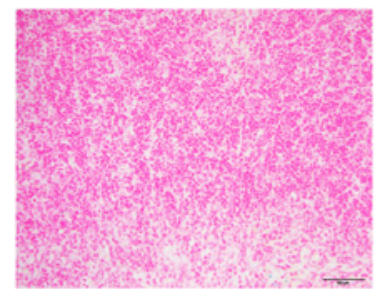

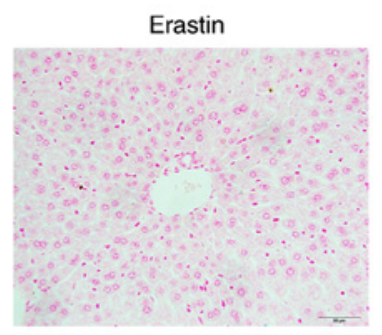

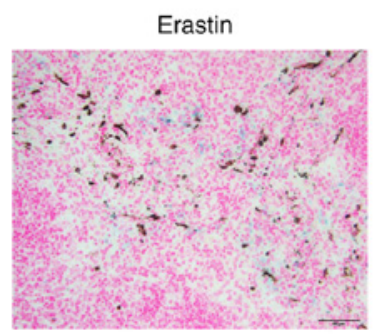

Figure 5. Erastin injection affects iron deposition. Representative images of hematoxylin-eosin staining in (A) liver and (C) spleen. Prussian blue staining in (B) liver and (D) spleen of control and erastin-treated groups (all magnifications, $\mathrm{x} 400$ ).

(Fig. 5D). In addition, erastin injection also caused severe iron deposition in the spleen (Fig. 5D).

\section{Discussion}

Ferroptosis is a recently defined programmed cell death, which has been revealed in various organ diseases, such as neurological disorders (16), liver injury (17) and cardiomyopathy (18). Ferroptosis is an iron-dependent non-apoptotic cell death that can produced by pharmacologically inhibiting the cysteine/glutamate antiporter, system $\mathrm{Xc}^{-}$or directly binding and loss of activity of GPX4 in cancer cells with high level RAS-RAF-MEK pathway activity or p53 expression, but it does not occur in normal cells (19). A series of small molecules 
including erastin have been found to induce ferroptosis in various cancer cells. They offer the possibility of cancer therapies through pharmacological interference with ferroptotic cell death, which has aroused great interest in scientific and medical researches (13). However, few studies focused on the effects of erastin-induced ferroptosis on healthy tissues and organs in vivo. The present study provided some evidences of metabolic and physiologic changes of mice injected with erastin.

According to the pharmacokinetics of erastin, erastin has low solubility and a previous study demonstrated a poor metabolic stability in a mouse liver microsome assay (4). The once-per-day-dosage frequency might be too low to see a tumor shrinking effect. Consequently, the present study conducted a preliminary in vivo study and selected a 2-day erastin administration protocol. Following intraperitoneal injection of erastin for 2 days, ferroptosis was induced, as expected, with accumulation of iron and lipid peroxides (Fig. 1). Erastin-induced ferroptosis caused the reduction of typical red-blood-cell-related hematological parameters, including red blood cell count, hematocrit, hemoglobin and red blood cell distribution width (Table I). This observation leads to the hypothesis that erastin tended to lead to anemia in mice. Alternation of blood indexes caused by erastin-induced ferroptosis was specific to red blood cells, but not platelets. In addition, increased serum iron and tissue iron were observed in the erastin-treated group. This suggested that erastin-caused anemia was not related to iron deficiency. This result may also hint at a targeted role of erastin to induce ferroptosis of erythrocytes. It has been reported that acute ingestion of large amounts of red blood cells could direct splenic macrophages to ferroptosis (20). Heme could mediate the activation and death of human platelets through ferroptosis (21). Iron constitutes the crucial part of heme and $\sim 70 \%$ of the body's iron is existed in red blood cells (22). It might be the reason that red blood cells, macrophages after robust erythrophagocytosis and platelets toxified by free heme are more sensitive to ferroptosis, which is an iron-dependent form of regulated cell death (1).

In its mouse model of erastin-induced ferroptosis, the present study observed mild infraction and iron deposition in the brain (Fig. 2), which was consistent with a previous study on cerebral ischemic/reperfusion (23). Due to the presence of the blood-brain barrier, the brain can adjust its iron status somewhat independently of other organs (24). For the low iron content in mouse brains, the classic Prussian blue staining had weak sensitivity for assessing iron content (24). The tiny blue granules observed in the cerebral sections may reflect a change of iron level in the brain of erastin-treated mice.

Ferroptosis is observed in the intestine of ischemia/reperfusion (8), ulcerative colitis (25) and cystic fibrosis (26). These studies explore the relationship between ferroptosis and the intestine in a pathological environment, but not in a healthy physiological state. The present study is a clear demonstration of the role of ferroptosis in duodenum growth as the villi were much stronger following erastin treatment (Fig. 3), which may be attributed to improved absorption of nutrients and shortened division cycle of intestinal stem cells. This result may once again suggest that ferroptosis was a double-edged sword in promoting and damaging organ growth. It has been reported that ferroptosis might be involved in limb development by helping remove interdigital webbing (27). Prussian blue staining also revealed that the blue granules of hemosiderin mainly appeared on the tip of villi in the duodenum of erastin-treated mice (Fig. 3E). It indicated that iron levels were increased in the epithelial cells of duodenum. Another possible explanation for ferroptosis leading to duodenum growth is that adequate iron supplementation may promote intestine growth, as iron supplementation increases villus height and depth, which is beneficial to intestinal mucosa in piglets (28).

Unexpectedly, the present study found that ferroptosis did not affect hepatic morphology or iron deposition in the mouse model of ferroptosis (Fig. 5). This result was inconsistent with previous report that ferroptosis was associated with liver injury (29), liver fibrosis (30) and nonalcoholic steatohepatitis (31). These differences might be attributed to the short-term injection of erastin. Notably, severe iron deposition and unknown substance accumulation were seen in the spleen of erastin-treated mice (Fig. 5). Ferroptosis can act on splenic macrophages (20) and cause DNA damage (32), but there was no deposition of unknown matter. Therefore, it was hypothesized that this substance might be secreted by the splenocytes, macrophages or other cells in the spleen, which needs further investigation.

In summary, the present study confirmed that erastin could induce ferroptosis in mice and revealed that erastin-induced ferroptosis altered the blood index values, causing mild cerebral infarction of brain and enlarged glomerular volume of kidney. Erastin also promoted the growth of duodenal epithelium with thicker, longer and denser villi in treated mice, but had no effect on liver or testis. These findings provided a new perspective for studying the role of erastin under healthy physiological conditions.

\section{Acknowledgements}

Not applicable.

\section{Funding}

This work was supported by Natural Science Foundation of Zhejiang province of China (no. LZ20C170004, LY19HD70003) and National Natural Science Foundation of China (no. 31872363).

\section{Availability of data and materials}

The datasets used and/or analyzed during the current study are available from the corresponding author on reasonable request.

\section{Authors' contributions}

$\mathrm{JZ}$ and HD were responsible for the conception and design of the study. JZ, BX and QX performed the experiments. JZ and $\mathrm{BX}$ confirm the authenticity of all the raw data. JZ, YF and HD performed the statistical analysis. YF and HD were responsible for funding acquisition. HD was responsible for project administration. JZ wrote the original draft. YF and HD wrote, reviewed and edited the manuscript. All authors read and approved the final manuscript. 


\section{Ethics approval and consent to participate}

The experimental procedures for the mice were approved by Animal Ethics Committee of Zhejiang University (approval no. 20077).

\section{Patient consent for publication}

Not applicable.

\section{Competing interests}

The authors declare that they have no competing interests.

\section{References}

1. Dixon SJ, Lemberg KM, Lamprecht MR, Skouta R, Zaitsev EM, Gleason CE, Patel DN, Bauer AJ, Cantley AM, Yang WS, et al: Ferroptosis: An iron-dependent form of nonapoptotic cell death. Cell 149: 1060-1072, 2012.

2. Tang D, Kang R, Berghe TV, Vandenabeele P and Kroemer G: The molecular machinery of regulated cell death. Cell Res 29: 347-364, 2019.

3. Xie Y, Hou W, Song X, Yu Y, Huang J, Sun X, Kang R and Tang D: Ferroptosis: Process and function. Cell Death Differ 23 369-379, 2016

4. Yang WS, SriRamaratnam R, Welsch ME, Shimada K, Skouta R, Viswanathan VS, Cheah JH, Clemons PA, Shamji AF, Clish CB, et al: Regulation of ferroptotic cancer cell death by GPX4. Cell 156: 317-331, 2014.

5. Xia Y, Sun X, Luo Y and Stary CM: Ferroptosis contributes to isoflurane neurotoxicity. Front Mol Neurosci 11: 486, 2019.

6. Belavgeni A, Meyer C, Stumpf J, Hugo C and Linkermann A: Ferroptosis and necroptosis in the kidneys. Cell Chem Biol 27: 448-462, 2020.

7. Yamada N, Karasawa T, Kimura H, Watanabe S, Komada T, Kamata R, Sampilvanjil A, Ito J, Nakagawa K, Kuwata H, et al: Ferroptosis driven by radical oxidation of $n-6$ polyunsaturated fatty acids mediates acetaminophen-induced acute liver failure. Cell Death Dis 11: 144, 2020.

8. Li Y, Feng D, Wang Z, Zhao Y, Sun R, Tian D, Liu D, Zhang F, Ning S, Yao J and Tian X: Ischemia-induced ACSL4 activation contributes to ferroptosis-mediated tissue injury in intestinal ischemia/reperfusion. Cell Death Differ 26: 2284-2299, 2019.

9. Dolma S,Lessnick SL, Hahn WC and Stockwell BR: Identification of genotype-selective antitumor agents using synthetic lethal chemical screening in engineered human tumor cells. Cancer Cell 3: 285-296, 2003

10. Bridges RJ, Natale NR and Patel SA: System $x^{-}$cystine/glutamate antiporter: An update on molecular pharmacology and roles within the CNS. Br J Pharmacol 165: 20-34, 2012.

11. Forcina GC and Dixon SJ: GPX4 at the crossroads of lipid homeostasis and ferroptosis. Proteomics 19: e1800311, 2019.

12. Xu T, Ding W, Ji X, Ao X, Liu Y, Yu W and Wang J: Molecular mechanisms of ferroptosis and its role in cancer therapy. J Cell Mol Med 23: 4900-4912, 2019.

13. Zhao Y, Li Y, Zhang R, Wang F, Wang T and Jiao Y: The role of erastin in ferroptosis and its prospects in cancer therapy. Onco Targets Ther 13: 5429-5441, 2020.

14. Huo H, Zhou Z, Qin J, Liu W, Wang B and Gu Y: Erastin disrupts mitochondrial permeability transition pore (mPTP) and induces apoptotic death of colorectal cancer cells. PLoS One 11: e0154605, 2016

15. Livak KJ and Schmittgen TD: Analysis of relative gene expression data using real-time quantitative PCR and the 2(-Delta Delta C(T)) method. Methods 25: 402-408, 2001.
16. Hambright WS, Fonseca RS, Chen L, Na R and Ran Q: Ablation of ferroptosis regulator glutathione peroxidase 4 in forebrain neurons promotes cognitive impairment and neurodegeneration. Redox Biol 12: 8-17, 2017.

17. Sui M, Jiang X, Chen J, Yang H and Zhu Y: Magnesium isoglycyrrhizinate ameliorates liver fibrosis and hepatic stellate cell activation by regulating ferroptosis signaling pathway. Biomed Pharmacother 106: 125-133, 2018.

18. Fang X, Wang H, Han D, Xie E, Yang X, Wei J, Gu S, Gao F, Zhu N, Yin X, et al: Ferroptosis as a target for protection against cardiomyopathy. Proc Natl Acad Sci USA 116: 2672-2680, 2019.

19. Imai H, Matsuoka M, Kumagai T, Sakamoto T and Koumura T: Lipid peroxidation-dependent cell death regulated by GPx4 and ferroptosis. Curr Top Microbiol Immunol 403: 143-170, 2017.

20. Youssef LA, Rebbaa A, Pampou S, Weisberg SP, Stockwell BR, Hod EA and Spitalnik SL: Increased erythrophagocytosis induces ferroptosis in red pulp macrophages in a mouse model of transfusion. Blood 131: 2581-2593, 2018.

21. NaveenKumar SK, SharathBabu BN, Hemshekhar M, Kemparaju K, Girish KS and Mugesh G: The role of reactive oxygen species and ferroptosis in heme-mediated activation of human platelets. ACS Chem Biol 13: 1996-2002, 2018.

22. Li X, He T, Yu K, Lu Q, Alkasir R, Guo G and Xue Y: Markers of iron status are associated with risk of hyperuricemia among Chinese adults: Nationwide population-based study. Nutrients 10: 191, 2018.

23. Tuo QZ, Lei P, Jackman KA, Li XL, Xiong H, Li XL, Liuyang ZY, Roisman L, Zhang ST, Ayton S, et al: Tau-mediated iron export prevents ferroptotic damage after ischemic stroke. Mol Psychiatry 22: 1520-1530, 2017

24. Hanninen MM, Haapasalo J, Haapasalo H, Fleming RE, Britton RS, Bacon BR and Parkkila S: Expression of iron-related genes in human brain and brain tumors. BMC Neurosci 10: 36 , 2009.

25. Xu M, Tao J, Yang Y, Tan S, Liu H, Jiang J, Zheng F and Wu B: Ferroptosis involves in intestinal epithelial cell death in ulcerative colitis. Cell Death Dis 11: 86, 2020.

26. Simões F, Ousingsawat J, Wanitchakool P, Fonseca A, Cabrita I, Benedetto R, Schreiber R and Kunzelmann K: CFTR supports cell death through ROS-dependent activation of TMEM16F (anoctamin 6). Pflugers Arch 470: 305-314, 2018.

27. Schnabel D, Salas-Vidal E, Narváez V, Sánchez-Carbente Mdel R, Hernández-García D, Cuervo R and Covarrubias L: Expression and regulation of antioxidant enzymes in the developing limb support a function of ROS in interdigital cell death. Dev Biol 291: 291-299, 2006.

28. Pu Y, Li S, Xiong H, Zhang X, Wang Y and Du H: Iron promotes intestinal development in neonatal piglets. Nutrients 10: 726, 2018.

29. Park SJ, Cho SS, Kim KM, Yang JH, Kim JH, Jeong EH, Yang JW, Han CY, Ku SK, Cho IJ and Ki SH: Protective effect of sestrin2 against iron overload and ferroptosis-induced liver injury. Toxicol Appl Pharmacol 379: 114665, 2019.

30. Kong Z, Liu R and Cheng Y: Artesunate alleviates liver fibrosis by regulating ferroptosis signaling pathway. Biomed Pharmacother 109: 2043-2053, 2019.

31. Tsurusaki S, Tsuchiya Y, Koumura T, Nakasone M, Sakamoto T, Matsuoka M, Imai H, Yuet-Yin Kok C, Okochi H, Nakano H, et al: Hepatic ferroptosis plays an important role as the trigger for initiating inflammation in nonalcoholic steatohepatitis. Cell Death Dis 10: 449, 2019.

32. Li T, Liu X, Jiang L, Manfredi J, Zha S and Gu W: Loss of p53-mediated cell-cycle arrest, senescence and apoptosis promotes genomic instability and premature aging. Oncotarget 7: $11838-11849,2016$

This work is licensed under a Creative Commons Attribution-NonCommercial-NoDerivatives 4.0 International (CC BY-NC-ND 4.0) License. 